\title{
A Contrastive Study of Chinese "Zhe" and English Present Continuous Tense
}

\author{
Jiali Cao \\ School of Foreign Languages, Guizhou Normal University, Guiyang, China \\ Email: 1843305521@qq.com
}

How to cite this paper: Cao, J. L. (2022). A Contrastive Study of Chinese "Zhe" and English Present Continuous Tense. Open Journal of Social Sciences, 10, 271-280. https://doi.org/10.4236/jss.2022.102019

Received: January 22, 2022

Accepted: February 21, 2022

Published: February 24, 2022

Copyright $\odot 2022$ by author(s) and Scientific Research Publishing Inc. This work is licensed under the Creative Commons Attribution-NonCommercial International License (CC BY-NC 4.0). http://creativecommons.org/licenses/by-nc/4.0/ (c) (i) $(9)$ Open Access

\begin{abstract}
In language system, Chinese is a typical inflectional language, while Chinese is one of the synthesis languages. There are some certain differences and similarities between Chinese and English in terms of language structure and grammatical meaning, etc. According to grammatical meaning, this article aims to analyze the corresponding relationship and certain differences between Chinese "zhe" and English present continuous tense. On the basis of theoretical analysis, it tries to offer some appropriate strategies for language teaching and learning.
\end{abstract}

\section{Keywords}

"Zhe", English Present Continuous Tense, Contrast, Teaching Strategy, Learning Strategy

\section{Introduction}

In recent years, our country's economy has been accelerating day by day, and its international status has been continuously improved. The influence of Chinese in the world has also been higher, and the climax of learning Chinese has been set off abroad gradually. However, Chinese belongs to the rare Sino-Tibetan languages, which also brings great difficulties to foreign learners because of its evident differences from the languages of other families. Based on previous research, this article starts with tense and analyzes the corresponding relationship and differences between Chinese "zhe" and English present continuous tense according to specific language materials. And it tries to match the grammatical categories and concepts of foreign languages in Chinese learning, and provides some appropriate strategies for language teaching and learning. In this article, its Chinese language materials mainly come from Corpus of Modern Chinese, and 
the English materials are mainly from British National Corpus (BNC) and Corpus of Contemporary English (COCA).

\section{Literature Review}

In China, the contrastive study between Chinese and other languages started relatively early. In recent decades of years, China's international influence has increased gradually and Chinese culture and language have received more and more admiration. The culture exchanges between China and foreign countries are deepening. More and more foreign students come to China to study Chinese. Therefore, the comparative and contrastive study between Chinese and English has been increasing day by day.

In terms of relative study and researches of Chinese "zhe", contemporary researches are mainly about the acquisition of "zhe" of foreign students. Xue (2003) has defined the grammatical meaning and categories of "zhe", and she investigated the situations of Korean students learning "zhe". Based on Chinese Intermediary Corpus System for foreign students, Gao (2013) counted the errors in the acquisition of "zhe" by European and American students, and she found that European and American students had a higher error rate generally. Liu (2009) expounded the different expressions of past, present and future tense in Chinese and English. Gao and Cao (2011) made a comprehensive and detailed review of the research results about "le", "zhe" and "guo" in home and abroad since 1980s. Zhang (2012) has investigated the errors of foreign language learners using dynamic auxiliary "zhe", and he also expounded their errors and analyzed relative reasons. Zhou (2013) explained the expressive forms and characteristics of tense in Chinese, English and French these three languages.

Based on previous research, this article takes tense as a starting point, contrasting and analyzing the differences and certain similarities between Chinese "zhe" and English present continuous tense, which aiming to offer some appropriate strategies for language teaching and learning.

\section{The Grammatical Meaning of Chinese "Zhe" and English Present Continuous Tense}

Chinese and English are from different language families. The former originates from Sino-Tibetan languages, while the latter belongs to Indo-European family. In their own language framework, both Chinese "zhe" and English present continuous tense have their own grammatical meaning respectively.

\subsection{The Grammatical Meaning of Chinese "Zhe"}

Chinese "zhe" is a hotspot in modern Chinese research, and many scholars have made relatively detailed and systematic elaboration of it. At present, most of scholars generally define Chinese "zhe" as a dynamic particle. In terms of dynamic particles, Huang and Liao (1979) pointed out that dynamic refers to the situation of an action or a character in the process of change, and it does not 
mean the time in which the action occurs, but it can represent the dynamics of time in the past, present or future. In terms of grammatical meaning, Zhu (1982) once stated that Chinese "zhe" means the continuation of an action or change and when it is used with fixed verbs, it means the continuity and progress of the action. In Modern Chinese Eight Hundred Words (Revised Edition), Lyu (1999) also included Chinese "zhe" into the auxiliary category, and he thought it was a dynamic auxiliary word that often follows verbs or adjectives without adding any modifiers in between, indicating an ongoing action or a continuing state. The grammatical meanings of Chinese "zhe" are roughly as follows:

A) Indicate the action that is in progress. For example:

1) Shang ban qian, fu qin kan zhe wu jian xin wen, ou er hai tai tou kan kan qiang shang de zhong biao. (上班前, 父亲看着午间新闻, 偶尔还抬头看看墙 上的钟表。)

2) Xia ban hui jia, yi da kai jia men, wo kan dao ye ye zheng zai ting zhe xiao qu er, nai nai zuo zai sha fa shang zhi zhe mao yi. (下班回家, 一打开家门, 我 看到爷爷正在听着小曲儿, 奶奶坐在沙发上织着毛衣。)

3) Xian sheng, jing li zheng kai zhe hui ne, ma fan nin zai xiu xi qu shao zuo deng hou. (先生, 经理正开着会呢, 麻烦您在休息区稍作等候。)

From the example sentences (1) - (3), it can be seen that the sentences with "zhe" generally indicate the action is taking place and it has lasted for a certain period of time. All these three sentences describe the daily communication. In example sentence (1), the speaker is going to school and his father is going to work, and they are in the lunch interval. It means that "kan xin wen" occurs in the present, and "zhe" is a description of the current progress of the action, which means that when the speaker sees his father, the action of "kan xin wen" is happening at that moment. Perhaps, outside of the father's interval time within the speaker, he is doing other things. Example sentence (2) is what "I" see when "I" reach home after work. "Zhe" is used after "ting", emphasizing that three actions of "da kai jia men", "ting xiao qu er" and "zhi mao yi" occur simultaneously. In example sentence (3), a father goes to the company to find his son who is a manager. The action of "kai hui" is continuous. It may be that when the speaker is talking, the manager is having a meeting, or the action of "kai hui" happened before "xian sheng" came and it has continued for a while.

B) Indicate the existential state of things in existential sentences. For example:

4) Shan gu li de xiao xi zheng chan chan di liu zhe. (山谷里的小溪正浔潺地 流着。)

5) Yao yao shu bao shang gua zhe de xiao ling dang fa chu qing cui de sheng yin. (瑶瑶书包上挂着的小铃铛发出清脆的声音。)

6) Ke ting zheng zhong jian bai zhe ba ba mai lai de xin dian shi ji. (客厅正中 间摆着爸爸买来的新电视机。)

Example sentence (4) describes a natural phenomenon. In this sentence, it means that the moment when the speaker sees the stream, the stream is flowing, 
which is the state of an ongoing action or existence of certain things. Example sentences (5) and (6) describe the daily phenomenon; "Yaoyao" bought a new bag with a little bell on it. "Gua zhe" and "bai zhe" indicate continuous state of things. After the actions of "bai zhe" and "gua zhe" are completed, the states of "ling dang" and "dian shi ji" do not change for a long time. Generally speaking, this kind of existence structure has two characteristics. The first is there is no "zai" and "zheng zai" before the verb, and the other is there is no complement or other dynamic auxiliary words before "zhe".

C) Indicate the continuation of the state after the previous action taking place. For example:

7) Mei gan de shang shang yi liang gong gong qi che, ta men zhi neng zuo zhe deng xia yi liang. (没赶得上上一辆公共汽车, 他们只能坐着等下一辆。)

8) Dao le feng shou shi jie le, lao nai nai pi zhe wai yi zuo zai chuang xia, zhu shi zhe chuang wai de tie da men, pan wang zhe xiao sun zi hui lai. (到了丰收 时节了, 老奶奶披着外衣坐在窗下, 注视着窗外的铁大门, 盼望着小孙子回 来。)

In example sentence (7), "ta men" are going to company by bus. "Zhe" is behind "zuo", that is, after the previous action "mei gan shang gong jiao che" occurs, the later action "sit" continues appropriately. In example (8), the son of "lao nai nai" is working in city, and "lao nai nai" misses him and her grandson very much. "Pan wang zhe" is used after "pi zhe" and "zhu shi zhe", indicating the continuation of the action in terms of the state. "Lao nai nai" has been expecting silently for a long time, and she is not doing other things absentmindedly.

D) "Zhe" is often combined with other verb phrases to express a certain state or a way. For example:

9) Ta ban zhe lian, jue zhe zui, di zhe tou jin le zheng wu. (他板着脸, 噘着 嘴, 低着头进了正屋。)

10) Wo men chang zhe tiao zhe, jiu zhe yang, ju hui zai da jia de huan xiao sheng zhong jie shu le. (我们唱着跳着, 就这样, 聚会在大家的欢笑声中结束 了。)

In the above two example sentences, both actions can be regarded as mutual accompaniment. In example (9), "ta" was not admitted by his ideal university. The three actions "ban zhe lian", "jue zhe zui" and "di zhe tou" are accompanied with each other. In example (10), the company held an annual meeting. "Chang zhe" and "tiao zhe" also mean that two actions occur at the same time. Both example sentences represent an existential state or a way.

\subsection{The Grammatical Meaning of English Present Continuous Tense}

Both Chinese and English are mainly divided into three major time intervals: past, present and future. English is an inflectional language, while Chinese is a synthetic language. There are certain evident differences between English and Chinese. However, some English tenses correspond to Chinese certain characters 
and they have obvious similarities. In terms of grammatical meaning, Chinese "zhe" is similar with English present continuous tense. By analyzing the differences and similarities between "zhe" and English present continuous tense, it can offer some appropriate suggestions and strategies for language learning.

A) Indicate an ongoing action, emphasizing action's instantaneousness, and it is often used with "now", "at this/that moment", etc. For example:

11) When I came back, my younger brother is reading an English book.

12) I am playing computer games now, while my mother is cooking.

Example (11) describes the situation when "I" come home from school. Example (12) describes the weekend life of "my" family. "Is reading" and "is cooking" both emphasize the instantaneity of the action rather than the continuity. In example (11), it is emphasized that "my younger brother" reads an English book at the moment "I" came back, and may not have read it before "I" came back. In example (12), it also emphasized that during the time "I" play computer games, "my mother" is cooking. Perhaps, "my mother" is doing other things outside of this period of time. Therefore, the English present continuous tense indicates the ongoing action.

B) Indicate the future, which means the action will happen according to the plan or arrangement, and its occurrence possibility is relatively high. For example:

13) I am going to park in 10 o'clock.

14) Mary and I are having dinner tonight.

In example sentences (13) and (14), both are the English present continuous tense indicating future, and "am going" and "are having" are upcoming actions. There is small difference between two ways indicating future in English: English present continuous tense and auxiliary verb "will". The former is more likely to occur, and "the future" described in the present continuous tense is closer to the present.

C) Indicate recurring or habitual actions, and it contains speakers' emotions such as praise, dissatisfaction, disgust and regret, etc. For example:

15) The girl is always smiling happily.

16) My brother is always making the same mistake.

Example sentence (15) describes a very kind neighboring girl, and sentence (16) tells "my brother's" weak mathematical foundation. "Is smiling" and "making mistakes" are recurring habitual actions. In example sentence (15), the speaker obviously praises the "girl". In example (16), the speaker is dissatisfied with "brother". Both of these two sentences express the speaker's subjective emotions by using present continuous tense.

D) Indicate the development process of things. For example:

17) Winter has come. It's getting colder and colder.

18) The leaves on the tree are turning yellow.

Example sentences (17) and (18) describe the changes caused by weather change. "Winter gets colder" and "leaves turn yellow" have to go through a stage 
or a period, and they can't change in an instant. Both of them belong to the development process of things.

\section{The Correspond Relationship and Differences}

Through above analysis, it can be seen that there is correspond relationship and also some certain differences between Chinese "zhe" and English present continuous tense.

\subsection{The Correspond Relationship}

A) Both indicate the action that is in progress. For example:

19) My father is watching TV.

Wo fu qin zheng zai kan zhe dian shi. (我父亲正在看着电视。)

20) Little cat is sleeping comfortably.

Xiao mao mi zheng shu fu di shui zhe. (小猫咪正舒服地睡着。)

Example sentences (19) and (20) describe daily situation of "my father" and "little cat". "Is watching" and "is sleeping" correspond to the Chinese "kan zhe" and "shui zhe" respectively, describing the action being performed at the moment. At this time, the meaning between Chinese "zhe" and English present continuous tense is overlapping.

B) Both indicate the continuation of the action or existential state after the action occurs. For example:

21) People are standing in supermarket to shelter from the rain.

Ren men zai chao shi li zhan zhe bi yu. (人们在超市里站着避雨。)

22) It's so hot. She is sitting under a tree to enjoy the cool.

Tian qi tai re le, ta zai shu xia zuo zhe cheng liang. (天气太热了, 他在树下坐 着乘凉。)

Example sentences (21) and (22) are people's response when they suffer the extreme weather situation. The rain will not stop immediately, and the weather will not turn cold in an instant, so "are standing" and "is sitting" both indicate states, corresponding with "zhan zhe" and "zuo zhe" in Chinese.

\subsection{Some Certain Differences}

A) English present continuous tense can express the process of the development of things, but Chinese "zhe" does not have the usage. For example:

23) It's getting dark.

Tian jian jian an xi lai. (天渐渐暗下来。)

24) The water is getting hot.

Shui man man bian re le. (水慢慢变热了。)

Both example sentences (23) and (24) are the natural phenomenon. "Is getting dark" and "is getting hot" are the process of the development of things, but there is no "zhe" in Chinese when we express this situation in Chinese. This is an obvious difference between Chinese "zhe" and English present continuous tense.

B) English present continuous can indicate the future, while there is no 
such usage of Chinese "zhe". For example:

25) We are going shopping next week.

Xia zhou wo men jiang qu gou wu. (下周我们将去购物。)

26) She is watching the competition tomorrow.

Ta ming tian jiang qu guan kan zhe chang bi sai. (她明天将去观看这场比 赛。)

Example sentences (25) and (26) are the things that are to happen according to appointment. It is obvious that English present continuous tense is used to indicate the future. But Chinese "zhe" does not have this usage. It is an obvious difference between Chinese "zhe" and English present continuous tense.

C) English present continuous tense can express subjective mood such as praise, dissatisfaction together with some certain adverbs like "forever/usually/always", etc, while Chinese "zhe" does not have the usage. For example:

27) Why are you always complaining?

Ni wei shen mo zong shi bao yuan ne? (你为什么总是抱怨呢?)

28) She is often encouraging others.

Ta jing chang gu li bie ren. (她经常鼓励别人。)

Example sentence (27) expresses speaker's attitude towards his friend when he quite dissatisfies and complains with the working environment. Example (28) is speaker's attitude to his English teacher. It can be seen that English present continuous tense can express speaker's subjective emotions. In example sentence (27), speaker expresses some dissatisfaction towards "you". In example sentence (28), speaker quite praises "she". But in Chinese, "zhe" does not have the usage.

\section{Teaching and Learning Strategy}

In terms of language learning, students are the major subjects and the most important or crucial factor. And teachers are also important external factors and they use effective and efficient ways to guide students' learning like a lighthouse. Therefore, both teachers and students should apply correct ways in language learning.

\subsection{Teacher's Teaching Strategy}

Both Chinese "zhe" and English present continuous are the grammatical content that are commonly used and easy to master under their own language frameworks. When teaching students, no matter what tense they are targeting, teachers should explain their basic meaning or relative usages first. That is to say, teachers should let students have a certain understanding of the basic usage of each tense, then add other usages and train some relatively complex sentence patterns gradually. Therefore, teachers should pay more attention to use a step-by-step method to guide students to master the knowledge. With the gradual accumulation of grammar knowledge, student's comprehension and acceptance ability will also be improved, and they will master the difficult but impor- 
tant points easily.

Secondly, teachers are supposed use the situational-teaching method to consolidate and practice repeatedly, strengthening the training of specific language knowledge. For foreign students, their ultimate goal is to be able to speak Chinese as well as having daily but normal communication. Therefore, teachers should look for relative topics from students' daily life after course-teaching, choosing the situations that students are familiar and set up a lot of language situations to stimulate students having situational dialogues. After class, it is also necessary for teachers to encourage students to find sentences in their daily life, strengthening the practice of specific language knowledge in practical application continuously, which can fully mobilize their enthusiasm for learning. In his way, teacher's teaching activities will be smoother, and students' learning efficiency will also be much higher.

Last but not least, interest is the best teacher. Students' personal emotion towards language also affects their learning efficiency to a certain extent. For example, some foreign students come to China to study Chinese because they love Chinese culture and language. Therefore, they have more interest and curiosity towards language learning, so they are willing to spending more time and energy learning and reviewing it. There is no doubt that they study harder than other students, and they will master certain language knowledge quickly. It is relatively easy for them to adjust to new environment, which plays an important role in learning language. By contrast, some other students always in a passive state in the new environment and knowledge. They accept the knowledge mechanically and seldom think and learn positively, so they are inclined to making errors. Teachers is significant in students' learning process, and they must encourage students and develop their interest and curiosity of learning language. They can use Chinese diverse culture to attract students' attention and interest.

\subsection{Student's Learning Strategy}

Generally speaking, it is more difficult for foreign students to learn Chinese than for Chinese students to learn foreign languages. Therefore, especially for foreign students, they themselves must be careful, learning to observe carefully to look for norms and rules in teacher's example sentences and homework exercises. In daily study, it is necessary to strengthen listening, speaking, reading and writing exercises and learn to draw and summarize rules and norms in learning.

In addition to study, they should communicate with local teachers and students about their learning experiences, difficulties and confusion. More importantly, foreign students also try to have more oral conversation with local students and discover their own shortcomings in time. And they must correct by themselves. Speaking is very important in learning languages. So foreign students must learn to "open their months" after the course. In addition, learning a kind of language will encounter different and various difficulties. Students have to have self-confidence and enthusiasm for learning. Even if encounter difficul- 
ties, they should not be discouraged. What they should do is to be confident and have methods to solve and deal with different problems correctly.

In the end, they must try their best to develop the interest of language learning. They can be familiar with Chinese or western culture firstly and then have a command of the language gradually. Interest is the best teacher. They need to learn language efficiently based on their own interest.

\section{Practical Implication on Teaching Practices}

Based on above analysis, it is obvious that there are evident correspond relationship and differences between Chinese "zhe" and English present continuous tense. Therefore, On the basis of similarities and differences, it can provide effective teaching methods and ways for teachers and students' learning in terms of different languages. Teachers can use comparative approach in teaching process. Only by mastering the differences and relative similarities between two languages, can students gradually summarize the rules or norms and become handy in language learning.

\section{Conclusion}

The article makes a contrastive analysis on Chinese "zhe" and English present continuous tense from the perspective of grammatical meaning based on specific language materials. It finds certain commonalities and differences between "zhe" and English present continuous tense in terms of grammatical meaning. In the end, it provides relatively effective suggestions and methods for teacher's teaching and student's learning, especially for foreign language teaching and learning. Teachers had better use step-by-step methods to guide students to master the language knowledge, and they also should be good at finding language materials after class. As the major subjects in learning process, students have to "open month" to communicate with local students and teachers. And they must be careful and confident enough to solve different difficulties and problems during language learning. Last but not least, they have to be relatively familiar with local culture of target language, and then develop interest and curiosity of language learning gradually.

\section{Conflicts of Interest}

The author declares no conflicts of interest regarding the publication of this paper.

\section{References}

Gao, R. (2013). A Study on the Acquisition of "De", "Zhe" and "Guo" in European and American Students. Master Dissertation, Northeast Normal University.

Gao, X., \& Cao, X. (2011). A Review of the Relevant Research of Modern Chinese "Le", "Zhe" and "Guo". Journal of Chuxiong Normal University.

Huang, B., \& Liao, X. (1979). The Modern Chinese. Higher Education Press. 
Liu, Y. (2009). On the Contrast of Tense Expression Means in Chinese, English and Chinese. Journal of Successful Education.

Lyv, S. (1999). Modern Chinese Eight Hundred Words (Revised ed.). The Commercial Press.

Xue, J. (2003). A Study on the Teaching of Modern Chinese Dynamic Auxiliary Words "Le", "Zhe" and "Guo" in Korea. Master Dissertation, Guangxi University.

Zhang, Y. (2012). Error Analysis of Dynamic Auxiliary Words "Le", "Zhe" and "Guo" and a Summary of Research on TCSL. Master Dissertation, Heilongjiang University.

Zhou, J. (2013). A Comparative Analysis of the Characteristics of Verb Tenses in Chinese, English and French. Journal of Yunyang Normal College.

Zhu, D. (1982). Grammar Notes. The Commercial Press. 